\title{
Developing Safe and Effective Devices for Neonatal Intensive Care
}

$\mathrm{N}$ eonatal intensive care units (NICU) depend heavily on biomedical devices and instruments for monitoring, diagnosis, and treatment. However, few such devices undergo meticulous research or rigorous testing. Developing safe and effective devices requires collaboration among diverse group of experts. To initiate such a process, the Eunice Kennedy Shriver National Institute of Child Health and Human Development (NICHD) invited an expert panel from biomedical and bioengineering disciplines for a workshop in 2009. The proceedings have been published (1); and a summary of the four topic areas discussed is presented below.

\section{CARDIOPULMONARY CARE AND MONITORING}

Many devices and instruments used for neonatal resuscitation in the delivery room need to be improved; these include methods to provide continuous or intermittent positive pressure; blenders to allow the use of variable inspired oxygen concentrations in the delivery rooms; and improved laryngoscopes to enable easy endotracheal (ET) intubation.

Similarly, ventilators used in NICU are to be developed specifically for use in newborn infants. All ventilators need improvement to minimize air leaks around the ET tubes; better high-frequency ventilators are needed for transportations; and better nasal interfaces are needed for continuous positive airway pressure and noninvasive ventilation. Similarly, simple techniques need to be developed to assess lung volumes and pulmonary mechanics continuously at the bedside. The ET tube technology also needs research for developing tubes with thinner and softer walls and larger inner diameters, preferably with inflatable cuffs (albeit intermittently) to help assess pulmonary functions.

Similarly, cardiac devices and instruments for measuring and monitoring blood pressure and cardiac output noninvasively and continuously, and methods to evaluate systemic oxygenation are urgently needed.

\section{CEREBROVASCULAR}

Because disturbed autoregulation of the cerebral blood flow may be the underlying physiologic variable for brain injury, there is a need to develop noninvasive hemodynamic monitors to assess the integrity of the cerebrovascular circulation. Such instruments would need to couple metabolic and perfusion data in a regionally specific manner.
The near-infrared spectroscopy (NIRS) technology needs to be improved to reduce motion artifacts, enhance regional specificity, and minimize measurement errors. Similarly, much research is needed in the new, light-emitting, diode-based and optical imaging systems for their incorporation into clinical practice.

\section{METABOLIC MONITORING}

Using advanced sensor technology, devices need to be developed for monitoring blood glucose noninvasive and continuously. Accurate micro-pump devices are needed to deliver minute quantities of drugs (e.g. insulin) with precision. Sensors with noninvasive techniques need to be developed to provide measuring and/or monitoring concentrations of serum electrolytes and serum lactate, among other biochemical parameters.

\section{NEONATAL SEPSIS}

Developing small, sensitive, practical, and robust sensors for detecting small numbers of microorganisms rapidly (with antibiotic sensitivity) at the point-of-care is urgently required. Biomarkers are to be developed to differentiate systemic infections from systemic inflammatory responses. Improved gloves and hand washing agents are needed for environmental asepsis and prevention of healthcareassociated infections. Tubes and catheters resistant to thrombus and bio-film formation (that may be coated with antibiotics or antiseptics) can help minimize catheter-related and health-care associated infections.

\section{SUMMARY}

The workshop panel identified many challenges in developing, testing, and marketing products for use in the NICU (1). However, it was optimistic that progress can be achieved through creative collaboration between the rapidly advancing bioengineering fields and biomedical disciplines. - Tonse N. K. Raju

\section{REFERENCE}

1. Raju TN, Stevenson DK, Higgins RD, Stark AR 2009 Safe and effective devices and
instruments for use in the neonatal intensive care units: NICHD Workshop summary. Biomed Instrum Technol 43:408-418 